УДК 512.5

T. O. Banakh, V. M. Gavrylkiv

\title{
AUTOMORPHISM GROUPS OF SUPEREXTENSIONS OF GROUPS
}

\begin{abstract}
T. O. Banakh, V. M. Gavrylkiv. Automorphism groups of superextensions of groups, Mat. Stud. 48 (2017), 134-142.

A family $\mathcal{L}$ of subsets of a set $X$ is called linked if $A \cap B \neq \varnothing$ for all $A, B \in \mathcal{L}$. A linked family $\mathcal{M}$ is maximal linked if $\mathcal{M}$ coincides with each linked family $\mathcal{L}$ on $X$ that contains $\mathcal{M}$. The superextension $\lambda(X)$ consists of all maximal linked families on $X$. Any associative binary operation $*: X \times X \rightarrow X$ can be extended to an associative binary operation $* \operatorname{colon} \lambda(X) \times$ $\lambda(X) \rightarrow \lambda(X)$. In the paper we study isomorphisms of superextensions of groups and prove that two groups are isomorphic if and only if their superextensions are isomorphic. Also we describe the automorphism groups of superextensions of all groups of order $\leq 5$.
\end{abstract}

Introduction. In this paper we investigate the automorphism group of the superextension $\lambda(G)$ of a group $G$. The thorough study of various extensions of semigroups was started in [12] and continued in [1]-[9], [13]-[17]. The largest among these extensions is the semigroup $v(S)$ of all upfamilies on $S$. A family $\mathcal{A}$ of non-empty subsets of a set $X$ is called an upfamily if for each set $A \in \mathcal{A}$ any subset $B \supset A$ of $X$ belongs to $\mathcal{A}$. Each family $\mathcal{B}$ of non-empty subsets of $X$ generates the upfamily $\langle B \subset X: B \in \mathcal{B}\rangle=\{A \subset X: \exists B \in \mathcal{B}(B \subset A)\}$. An upfamily $\mathcal{F}$ that is closed under taking finite intersections is called a filter. A filter $\mathcal{U}$ is called an ultrafilter if $\mathcal{U}=\mathcal{F}$ for any filter $\mathcal{F}$ containing $\mathcal{U}$. The family $\beta(X)$ of all ultrafilters on a set $X$ is called the Stone-Čech compactification of $X$, see [19], [23]. An ultrafilter, generated by a singleton $\{x\}, x \in X$, is called principal. Each point $x \in X$ is identified with the principal ultrafilter $\langle\{x\}\rangle$ generated by the singleton $\{x\}$, and hence we can consider $X \subset \beta(X) \subset v(X)$. It was shown in [12] that any associative binary operation $*$ colon $S \times S \rightarrow S$ can be extended to an associative binary operation $*: v(S) \times v(S) \rightarrow v(S)$ defined by the formula

$$
\mathcal{L} * \mathcal{M}=\left\langle\bigcup_{a \in L} a * M_{a}: L \in \mathcal{L}, \quad\left\{M_{a}\right\}_{a \in L} \subset \mathcal{M}\right\rangle
$$

for upfamilies $\mathcal{L}, \mathcal{M} \in v(S)$. In this case the Stone-Čech compactification $\beta(S)$ is a subsemigroup of the semigroup $v(S)$.

The semigroup $v(S)$ contains many other important extensions of $S$. In particular, it contains the semigroup $\lambda(S)$ of maximal linked upfamilies. The space $\lambda(S)$ is well-known in General and Categorial Topology as the superextension of $S$, see [21]-[24]. An upfamily $\mathcal{L}$ of subsets of $S$ is linked if $A \cap B \neq \varnothing$ for all $A, B \in \mathcal{L}$. The family of all linked upfamilies on $S$ is denoted by $N_{2}(S)$. It is a subsemigroup of $v(S)$. The superextension $\lambda(S)$ consists of all maximal elements of $N_{2}(S)$, see [11], [12].

2010 Mathematics Subject Classification:20B25, 20D45, 20M15.

Keywords: group; semigroup; maximal linked family; superextension; automorphism group. doi:10.15330/ms.48.2.134-142

(C) T. O. Banakh, V. M. Gavrylkiv, 2017 
For a finite set $X$, the cardinality of the set $\lambda(X)$ grows very quickly as $|X|$ tends to infinity. The calculation of the cardinality of $\lambda(X)$ seems to be a difficult combinatorial problem, which can be reformulated as the problem of counting the number $\lambda(n)$ of self-dual monotone Boolean functions of $n$ variables, which is well-known in Discrete Mathematics. According to Proposition 1.1 in [10], $\log _{2} \lambda(n)=\frac{2^{n}}{\sqrt{2 \pi n}}+o(1)$, which means that the sequence $(\lambda(n))_{n=1}^{\infty}$ has double exponential growth. The sequence of numbers $\lambda(n)$ (known in Discrete Mathematics as Hoşten-Morris numbers) is included in the On-line Encyclopedia of Integer Sequences as the sequence A001206. All known precise values of this sequence (taken from [10]) are presented in the following table.

\begin{tabular}{|r|ccccccccc|}
\hline$|X|=$ & 1 & 2 & 3 & 4 & 5 & 6 & 7 & 8 & 9 \\
\hline$|\lambda(X)|=$ & 1 & 2 & 4 & 12 & 81 & 2646 & 1422564 & 229809982112 & 423295099074735261880 \\
\hline
\end{tabular}

Each map $f: X \rightarrow Y$ induces the map $\lambda f: \lambda(X) \rightarrow \lambda(Y), \lambda f: \mathcal{M} \mapsto\langle f(M) \subset$ $Y: M \in \mathcal{M}\rangle$, see [11].

If $\varphi: S \rightarrow S^{\prime}$ is a homomorphism of semigroups, then $\lambda \varphi: \lambda(S) \rightarrow \lambda\left(S^{\prime}\right)$ is a homomorphism as well, see [14].

A non-empty subset $I$ of a semigroup $S$ is called an ideal if $I S \cup S I \subset I$. An ideal $I$ of a semigroup $S$ is said to be proper if $I \neq S$. A proper ideal $M$ of $S$ is maximal if $M$ coincides with each proper ideal $I$ of $S$ that contains $M$.

An element $z$ of a semigroup $S$ is called a zero (resp. a left zero, a right zero) in $S$ if $a z=z a=z$ (resp. $z a=z, a z=z)$ for any $a \in S$. An element $e$ of a semigroup $S$ is called an idempotent if $e e=e$. By $E(S)$ we denote the set of all idempotents of a semigroup $S$.

Recall that an isomorphism between semigroups $S$ and $S^{\prime}$ is a bijective function $\psi: S \rightarrow$ $S^{\prime}$ such that $\psi(x y)=\psi(x) \psi(y)$ for all $x, y \in S$. If there exists an isomorphism between $S$ and $S^{\prime}$, then $S$ and $S^{\prime}$ are said to be isomorphic, denoted $S \cong S^{\prime}$. An isomorphism $\psi: S \rightarrow S$ is called an automorphism of a semigroup $S$. By Aut $(S)$ we denote the automorphism group of a semigroup $S$.

Following the algebraic tradition, we take for a model of a cyclic group of order $n$ the multiplicative group $C_{n}=\left\{z \in \mathbb{C}: z^{n}=1\right\}$ of $n$-th roots of 1 .

For a set $X$ by $S_{X}$ we denote the group of all bijections of $X$. For two sets $X \subset Y$ we shall identify $S_{X}$ with the subgroup $\left\{\varphi \in S_{Y}: \varphi \mid Y \backslash X=\right.$ id $\}$.

1. Extending isomorphisms from groups to their superextensions. In this section we observe that each isomorphism of groups can be extended to an isomorphism of their superextensions and two groups are isomorphic if and only if their superextensions are isomorphic. The following statements are corollaries of the functoriality of the superextension in the category of semigroups, see $[3,23]$.

Proposition 1. If $\psi: G \rightarrow H$ is an isomorphism of groups, then $\lambda \psi: \lambda(G) \rightarrow \lambda(H)$ is an isomorphism of their superextensions.

Corollary 1. If $\psi: G \rightarrow G$ is an automorphism of a group $G$, then $\lambda \psi: \lambda(G) \rightarrow \lambda(G)$ is an automorphism of the superextension $\lambda(G)$.

Corollary 2. The automorphism group Aut $(\lambda(G))$ of the superextension of a group $G$ contains as a subgroup an isomorphic copy of the automorphism group Aut $(G)$ of $G$. 
Corollary 2 motivates a question: is the automorphism group Aut $(G)$ of a group $G$ normal in the automorphism group $\operatorname{Aut}(\lambda(G))$ of its superextension $\lambda(G)$ ? In the next section we show that the automorphism group Aut $\left(C_{2} \times C_{2}\right)$ of the Klein four-group $C_{2} \times C_{2}$ is not normal in $\operatorname{Aut}\left(\lambda\left(C_{2} \times C_{2}\right)\right)$.

Proposition 2. Let $G$ and $H$ be groups. If $\psi: \lambda(G) \rightarrow \lambda(H)$ is an isomorphism, then $\psi(G)=H$.

Proof. It was shown in [1, Proposition 1.1] that $\lambda(G) \backslash G$ is an ideal of $\lambda(G)$. Let us prove that $\lambda(G) \backslash G$ is the unique maximal ideal of $\lambda(G)$. Indeed, let $I$ be any ideal of $\lambda(G)$. If $g \in G \cap I$, then $\lambda(G)=g \lambda(G) \subset I$, and hence $I=\lambda(G)$. Consequently, $\lambda(G) \backslash G$ contains each proper ideal of $\lambda(G)$. In the same way $\lambda(H) \backslash H$ is the unique maximal ideal of $\lambda(H)$. Taking into account that the set of maximal ideals of a semigroup is preserved by isomorphisms and $\lambda(G) \backslash G$ and $\lambda(H) \backslash H$ are unique maximal ideals of $\lambda(G)$ and $\lambda(H)$ respectively, we conclude that $\psi(\lambda(G) \backslash G)=\lambda(H) \backslash H$. Therefore, $\psi(G)=H$.

Corollary 3. For any groups $G$ and $H$, each isomorphism from $\lambda(G)$ to $\lambda(H)$ is an extension of an isomorphism from $G$ to $H$.

Corollary 4. For any group $G$, each automorphism of $\lambda(G)$ is an extension of an automorphism of $G$.

Propositions 1 and 2 imply the following theorem.

Theorem 1. Two groups are isomorphic if and only if their superextensions are isomorphic.

2. The automorphism groups of the superextensions of groups of order $\leq 5$. In this section we shall study automorphisms of superextensions of groups and describe the structure of the automorphism groups of superextensions $\lambda(G)$ of finite groups $G$ of cardinality $|G| \leq 5$.

Before describing the structure of extensions of finite groups, let us make some remarks concerning the structure of a semigroup $S$ containing a group $G$ with the identity element which also is a left identity of $S$. In this case $S$ can be thought as a $G$-space endowed with the left action of the group $G$. So we can consider the orbit space $S / G=\{G s: s \in S\}$ and the projection $\pi: S \rightarrow S / G$. If $G$ lies in the center of the semigroup $S$ (which means that the elements of $G$ commute with all the elements of $S$ ), then the orbit space $S / G$ admits a unique semigroup operation making $S / G$ a semigroup and the orbit projection $\pi: S \rightarrow S / G$ into a semigroup homomorphism. If $s \in S$ is an idempotent, then the orbit $G s$ is a group isomorphic to a quotient group of $G$. A subsemigroup $T \subset S$ will be called a transversal semigroup if the restriction $\pi: T \rightarrow S / G$ is an isomorphism of the semigroups. If $S$ admits a transversal semigroup $T$ and the elements of $G$ and $T$ commute, then $S$ is a homomorphic image of the product $G \times T$ under the semigroup homomorphism $h: G \times T \rightarrow S, \quad h:(g, t) \mapsto g t$. This helps to recover the algebraic structure of $S$ from the structure of a transversal semigroup.

First note that each group $G$ of cardinality $|G| \leq 5$ is Abelian and is isomorphic to one of the groups: $C_{1}, C_{2}, C_{3}, C_{4}, C_{2} \times C_{2}, C_{5}$.

2.1. The semigroups $\lambda\left(C_{1}\right)$ and $\lambda\left(C_{2}\right)$. For the groups $C_{n}$ with $n \in\{1,2\}$ the semigroup $\lambda\left(C_{n}\right)$ is isomorphic to $C_{n}$. Therefore, $\operatorname{Aut}\left(\lambda\left(C_{n}\right)\right) \cong \operatorname{Aut}\left(C_{n}\right) \cong C_{1}$.

2.2. The semigroup $\lambda\left(C_{3}\right)$. For the group $C_{3}$ the semigroup $\lambda\left(C_{3}\right)$ contains three principal ultrafilters $1, z,-z$ where $z=e^{2 \pi i / 3}$ and the maximal linked upfamily $\triangle=\langle\{1, z\},\{1,-z\}$, 
$\{z,-z\}\rangle$ which is the zero in $\lambda\left(C_{3}\right)$. The superextension $\lambda\left(C_{3}\right)$ is isomorphic to the multiplicative subsemigroup $\left\{z \in \mathbb{C}: z^{4}=z\right\}$ of the complex plane. Taking into account that the zero is preserved by automorphisms of semigroups, we conclude that each automorphism of $C_{3}$ is extended to the unique automorphism of $\lambda\left(C_{3}\right)$ by Corollary 4. Therefore, $\operatorname{Aut}\left(\lambda\left(C_{3}\right)\right) \cong \operatorname{Aut}\left(C_{3}\right) \cong C_{2}$.

2.3. The semigroup $\lambda\left(C_{4}\right)$. Consider the cyclic group $C_{4}=\{1,-1, i,-i\}$. The semigroup $\lambda\left(C_{4}\right)$ contains 12 elements $\lambda\left(C_{4}\right)=\left\{g, g \triangle, g \square: g \in C_{4}\right\}$, where

$$
\triangle=\langle\{1, i\},\{1,-i\},\{i,-i\}\rangle \text { and } \square=\langle\{1, i\},\{1,-i\},\{1,-1\},\{i,-i,-1\}\rangle .
$$

Taking into account that $\triangle * \square=\square * \triangle=\triangle, \triangle * \triangle=\square * \square=\square$ and $C_{4}$ lies in the center of $\lambda\left(C_{4}\right)$, we conclude that $\lambda\left(C_{4}\right)$ contains a transversal semigroup $T=\{1, \triangle, \square\}$, where 1 is the identity of $C_{4}$.

Let $\psi: \lambda\left(C_{4}\right) \rightarrow \lambda\left(C_{4}\right)$ be an automorphism. Then the restriction of $\psi$ to $C_{4}$ is an automorphism of $C_{4}$ by Proposition 2. Taking into account that $\square$ is the unique idempotent of $\lambda\left(C_{4}\right) \backslash C_{4}$, we conclude that $\psi(\square)=\square$, and hence $\psi(g \square)=\psi(g) * \psi(\square)=\psi(g) * \square$ for any $g \in C_{4}$. Let $\psi(\triangle)=a \triangle$ for some $a \in C_{4}$. Then

$$
\square=\psi(\square)=\psi(\triangle * \triangle)=\psi(\triangle) * \psi(\triangle)=a \triangle * a \triangle=a^{2} \square .
$$

So $a^{2}=1$ and $a \in\{1,-1\}$.

Since $\operatorname{Aut}\left(C_{4}\right) \cong C_{2}$ and each automorphism of $C_{4}$ can be can be extended to an automorphism of $\lambda\left(C_{4}\right)$ exactly in two different ways, the group Aut $\left(\lambda\left(C_{4}\right)\right)$ contains four elements. Taking into account that each non-identity element $\psi \in \operatorname{Aut}\left(\lambda\left(C_{4}\right)\right)$ has order 2 , we conclude that $\operatorname{Aut}\left(\lambda\left(C_{4}\right)\right) \cong C_{2} \times C_{2}$.

2.4. The semigroup $\lambda\left(C_{2} \times C_{2}\right)$. The semigroup $\lambda\left(C_{2} \times C_{2}\right)$ has a similar algebraic structure. It also contains 12 elements $\lambda\left(C_{2} \times C_{2}\right)=\left\{g, g \triangle, g \square: g \in C_{2} \times C_{2}\right\}$, where

$$
\begin{gathered}
\triangle=\langle\{(1,1),(1,-1)\},\{(1,1),(-1,1)\},\{(1,-1),(-1,1)\}\rangle, \\
\square=\langle\{(1,1),(1,-1)\},\{(1,1),(-1,1)\},\{(1,1),(-1,-1)\},\{(1,-1),(-1,1),(-1,-1)\}\rangle .
\end{gathered}
$$

Taking into account that $\triangle * \square=\square * \triangle=\triangle, \triangle * \triangle=\square * \square=\square$ and $C_{2} \times C_{2}$ lies in the center of $\lambda\left(C_{2} \times C_{2}\right)$, we conclude that $\lambda\left(C_{2} \times C_{2}\right)$ contains a transversal semigroup

$$
T=\{e, \triangle, \square\} \subset \lambda\left(C_{2} \times C_{2}\right),
$$

where $e$ is the principal ultrafilter supported by the neutral element $(1,1)$ of $C_{2} \times C_{2}$.

We shall prove that the automorphism group Aut $\left(\lambda\left(C_{2} \times C_{2}\right)\right)$ of the semigroup $\lambda\left(C_{2} \times C_{2}\right)$ is isomorphic to the holomorph $\mathrm{Hol}\left(C_{2} \times C_{2}\right)$ of the group $C_{2} \times C_{2}$.

We recall that the holomorph $\mathrm{Hol}(S)$ of a semigroup $S$ (see [22]) is the semi-direct product $S \rtimes \operatorname{Aut}(S):=(S \times \operatorname{Aut}(S), \star)$ of the semigroup $S$ with its automorphism group Aut $(S)$, endowed with the semigroup operation

$$
(x, f) \star(y, g)=(x \cdot f(y), f \circ g) .
$$

It is known ${ }^{1}$ that for the group $G=C_{2} \times C_{2}$ its holomorph $\mathrm{Hol}(G)$ is isomorphic to the symmetric group $S_{4}$.

https://groupprops.subwiki.org/wiki/Holomorph_of_a_group 
Proposition 3. For the group $G=C_{2} \times C_{2}$ the automorphism group $\operatorname{Aut}(\lambda(G))$ is isomorphic to the holomorph $\mathrm{Hol}(G)$ of the group $G$ and hence is isomorphic to the symmetric group $S_{4}$.

Proof. Let $\psi: \lambda(G) \rightarrow \lambda(G)$ be an automorphism. Then the restriction of $\psi$ to $G$ is an automorphism of $G$ by Proposition 2. Taking into account that $\square$ is the unique idempotent of $\lambda(G) \backslash G$, we conclude that $\psi(\square)=\square$ and $\psi(g \square)=\psi(g) * \psi(\square)=\psi(g) * \square$ for any $g \in G$. It follows that $\psi(\triangle)=a \triangle$ for some $a \in G$.

It can be shown that for any pair $(a, f) \in G \times \operatorname{Aut}(G)$ the map $\psi_{a, f}: \lambda\left(C_{2} \times C_{2}\right) \rightarrow$ $\lambda\left(C_{2} \times C_{2}\right)$ defined by $\psi_{a, f}(x)=f(x), \quad \psi_{a, f}(x \square)=f(x) \square, \quad \psi_{a, f}(x \triangle)=f(x) \cdot a \triangle$ for $x \in G$ is an automorphism of the semigroup $\lambda\left(C_{2} \times C_{2}\right)$.

It follows that each automorphism of $\lambda(G)$ is of the form $\psi_{a, f}$ for some $(a, f) \in G \times$ $\operatorname{Aut}(G)$.

Observe that for any $(a, f),(b, g) \in G \times \operatorname{Aut}(G)$ and $x \in G$ we get

$$
\begin{gathered}
\psi_{a, f} \circ \psi_{b, g}(x)=\psi_{a, f}(g(x))=f \circ g(x), \quad \psi_{a, f} \circ \psi_{b, g}(x \cdot \square)=\psi_{a, f}(g(x) \cdot \square)=f \circ g(x) \cdot \square, \\
\psi_{a, f} \circ \psi_{b, g}(x \cdot \triangle)=\psi_{a, f}(g(x) \cdot b \cdot \triangle)=f \circ g(x) \cdot f(b) \cdot a \cdot \triangle .
\end{gathered}
$$

Consequently, $\psi_{a, f} \circ \psi_{b, g}=\psi_{a \cdot f(b), f \circ g}$ and hence Aut $(\lambda(G))$ is isomorphic to the holomorph $\operatorname{Hol}(G)$ of the group $G$, which is known to be isomorphic to the symmetric group $S_{4}$.

2.5. The semigroup $\lambda\left(C_{5}\right)$. In this subsection we describe the structure of automorphism group of the semigroup $\lambda\left(C_{5}\right)$. The algebraic structure of the semigroup $\lambda\left(C_{5}\right)$ is described in [9]. This semigroup contains 81 elements. One of them is zero $\mathcal{Z}=\left\{A \subset C_{5}:|A| \geq 3\right\}$, which is invariant under any bijection of $C_{5}$. All the other 80 elements have 5-element orbits under the action of $C_{5}$, which implies that the orbit semigroup $\lambda\left(C_{5}\right) / C_{5}$ consists of 17 elements.

It will be convenient to think of $C_{5}$ as the field $\{0,1,2,3,4\}$ with the multiplicative subgroup $C_{5}^{*}=\{1,-1,2,-2\}$ of invertible elements (here -1 and -2 are identified with 4 and 3 , respectively). Also for elements $x, y, z \in C_{5}$ we shall write $x y z$ instead of $\{x, y, z\}$.

The semigroup $\lambda\left(C_{5}\right)$ contains 5 idempotents: $\mathcal{U}=\langle 0\rangle, \mathcal{Z}, \Lambda_{4}=\langle 01,02,03,04,1234\rangle$, $\Lambda=\langle 02,03,123,014,234\rangle, 2 \Lambda=\langle 04,01,124,023,143\rangle$, which commute and thus form a commutative subsemigroup $E\left(\lambda\left(C_{5}\right)\right)$. Being a semilattice, $E\left(\lambda\left(C_{5}\right)\right)$ carries a natural partial order: $e \leq f$ iff $e * f=e$. The partial order $\mathcal{Z} \leq \Lambda, 2 \Lambda \leq \Lambda_{4} \leq \mathcal{U}$ on the set $E\left(\lambda\left(C_{5}\right)\right)$ is drawn in the picture:

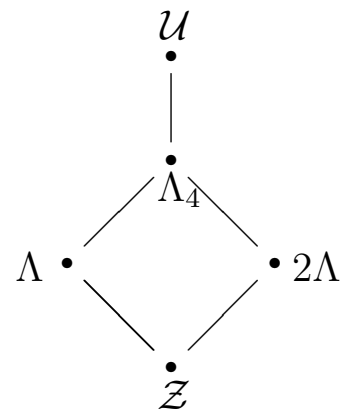

Diagram 1. The structure of the semilattice $E\left(\lambda\left(C_{5}\right)\right)$.

Next, consider two subsets: $\sqrt{\mathcal{Z}}=\left\{\mathcal{L} \in \lambda\left(C_{5}\right): \mathcal{L} * \mathcal{L}=\mathcal{Z}\right\}$ and $\sqrt{E\left(\lambda\left(C_{5}\right)\right)}=\left\{\mathcal{L} \in \lambda\left(C_{5}\right): \mathcal{L} * \mathcal{L} \in E\left(\lambda\left(C_{5}\right)\right)\right\}=\left\{\mathcal{L} \in \lambda\left(C_{5}\right): \mathcal{L} * \mathcal{L} * \mathcal{L} * \mathcal{L}=\mathcal{L} * \mathcal{L}\right\}$. 
We claim that the set $\sqrt{E\left(\lambda\left(C_{5}\right)\right)} \backslash \sqrt{\mathcal{Z}}$ has at most one-point intersection with each orbit. Indeed, if $\mathcal{L} \in \sqrt{E\left(\lambda\left(C_{5}\right)\right)}$ and $\mathcal{L} * \mathcal{L} \neq \mathcal{Z}$, then for every $a \in C_{5} \backslash\{0\}$, we get

$$
\begin{gathered}
(\mathcal{L}+a) *(\mathcal{L}+a) *(\mathcal{L}+a) *(\mathcal{L}+a)=\mathcal{L} * \mathcal{L} * \mathcal{L} * \mathcal{L}+4 a= \\
=\mathcal{L} * \mathcal{L}+4 a \neq \mathcal{L} * \mathcal{L}+2 a=(\mathcal{L}+a) *(\mathcal{L}+a)
\end{gathered}
$$

witnessing that $\mathcal{L}+a \notin \sqrt{E\left(\lambda\left(C_{5}\right)\right)}$.

By a direct calculation one can check that the set $\sqrt{E\left(\lambda\left(C_{5}\right)\right)}$ contains the following four maximal linked upfamilies:

$$
\begin{gathered}
\Delta=\langle 02,03,23\rangle, \quad \Lambda_{3}=\langle 02,03,04,234\rangle \\
\Theta=\langle 14,012,013,123,024,034,234\rangle, \quad \Gamma=\langle 02,04,013,124,234\rangle .
\end{gathered}
$$

For those upfamilies we get

$$
\begin{gathered}
\Delta * \Delta=\Delta * \Delta * \Delta=\Lambda, \quad \Lambda_{3} * \Lambda_{3}=\Lambda_{3} * \Lambda_{3} * \Lambda_{3}=\Lambda \\
\mathcal{F} * \Theta=\mathcal{F} * \Gamma=\mathcal{Z} \text { for every } \mathcal{F} \in \lambda\left(C_{5}\right) \backslash C_{5}
\end{gathered}
$$

All the other elements of $\lambda\left(C_{5}\right)$ can be found as images of $\Delta, \Theta, \Gamma, \Lambda_{3}$ under the affine transformations of the field $C_{5}$. Those are maps of the form $f_{a, b}: x \mapsto a x+b \bmod 5$, where $a \in\{1,-1,2,-2\}=C_{5}^{*}$ and $b \in C_{5}$. The image of a maximal linked upfamily $\mathcal{L} \in \lambda\left(C_{5}\right)$ under such a transformation will be denoted by $a \mathcal{L}+b$.

One can check that $a \Lambda_{4}=\Lambda_{4}$ for each $a \in C_{5}^{*}$ while $\Lambda=-\Lambda$, and $\Theta=-\Theta$. Since the linear transformations of the form $f_{a, 0}: C_{5} \rightarrow C_{5}, a \in C_{5}^{*}$, are authomorphisms of the group $C_{5}$, the induced transformations $\lambda f_{a, 0}: \lambda\left(C_{5}\right) \rightarrow \lambda\left(C_{5}\right)$ are authomorphisms of the semigroup $\lambda\left(C_{5}\right)$. This implies that those transformations do not move the subsets $E\left(\lambda\left(C_{5}\right)\right)$ and $\sqrt{E\left(\lambda\left(C_{5}\right)\right)}$. Consequently, the set $\sqrt{E\left(\lambda\left(C_{5}\right)\right.}$ contains the maximal linked upfamilies: $a \Delta, a \Theta, a \Lambda_{3}, a \Gamma, a \in \mathbb{Z}_{5}^{*}$, which together with the idempotents form a 17-element subset

$$
T_{17}=E\left(\lambda\left(C_{5}\right)\right) \cup\{a \Delta, a \Theta: a \in\{1,2\}\} \cup\left\{a \Lambda_{3}, a \Gamma: a \in \mathbb{Z}_{5}^{*}\right\}
$$

that projects bijectively onto the orbit semigroup $\lambda\left(C_{5}\right) / C_{5}$. The set $T_{17}$ looks as follows (we connect an element $x \in T_{17}$ with an idempotent $e \in T_{17}$ by an arrow if $x * x=e$ ):

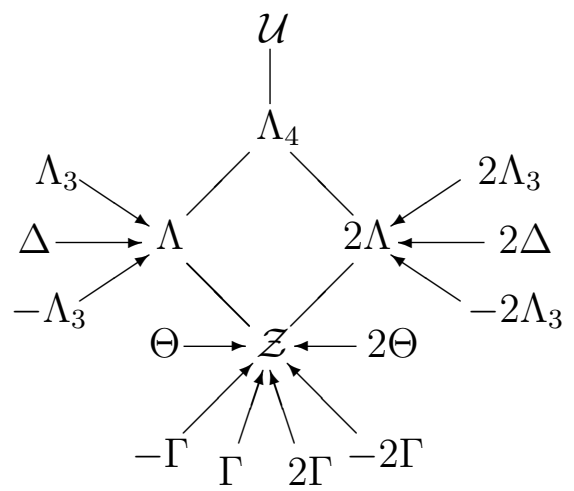

It follows that $\sqrt{E\left(\lambda\left(C_{5}\right)\right)}=T_{17} \cup \sqrt{\mathcal{Z}}$ where $\sqrt{\mathcal{Z}}=\{\Theta, 2 \Theta, \Gamma, 2 \Gamma,-\Gamma,-2 \Gamma\}+C_{5}$. 


\begin{tabular}{|c|c|cccc|cccc|c||}
\hline$*$ & $\Lambda_{4}$ & $\Lambda$ & $\Delta$ & $\Lambda_{3}$ & $-\Lambda_{3}$ & $2 \Lambda$ & $2 \Delta$ & $2 \Lambda_{3}$ & $-2 \Lambda_{3}$ & $a \Theta, a \Gamma$ \\
\hline \hline$\Lambda_{4}$ & $\Lambda_{4}$ & $\Lambda$ & $\Lambda$ & $\Lambda$ & $\Lambda$ & $2 \Lambda$ & $2 \Lambda$ & $2 \Lambda$ & $2 \Lambda$ & $\mathcal{Z}$ \\
\hline$\Lambda$ & $\Lambda$ & $\Lambda$ & $\Lambda$ & $\Lambda$ & $\Lambda$ & $\mathcal{Z}$ & $\mathcal{Z}$ & $\mathcal{Z}$ & $\mathcal{Z}$ & $\mathcal{Z}$ \\
\hline$\Delta$ & $\Delta$ & $\Lambda$ & $\Lambda$ & $\Lambda$ & $\Lambda$ & $2 \Theta$ & $2 \Theta$ & $2 \Theta$ & $2 \Theta$ & $\mathcal{Z}$ \\
$\Lambda_{3}$ & $\Lambda_{3}$ & $\Lambda$ & $\Lambda$ & $\Lambda$ & $\Lambda$ & $2 \Theta+2$ & $2 \Theta+2$ & $2 \Theta+2$ & $2 \Theta+2$ & $\mathcal{Z}$ \\
$-\Lambda_{3}$ & $-\Lambda_{3}$ & $\Lambda$ & $\Lambda$ & $\Lambda$ & $\Lambda$ & $2 \Theta-2$ & $2 \Theta-2$ & $2 \Theta-2$ & $2 \Theta-2$ & $\mathcal{Z}$ \\
\hline $2 \Lambda$ & $2 \Lambda$ & $\mathcal{Z}$ & $\mathcal{Z}$ & $\mathcal{Z}$ & $\mathcal{Z}$ & $2 \Lambda$ & $2 \Lambda$ & $2 \Lambda$ & $2 \Lambda$ & $\mathcal{Z}$ \\
\hline $2 \Delta$ & $2 \Delta$ & $\Theta$ & $\Theta$ & $\Theta$ & $\Theta$ & $2 \Lambda$ & $2 \Lambda$ & $2 \Lambda$ & $2 \Lambda$ & $\mathcal{Z}$ \\
$2 \Lambda_{3}$ & $2 \Lambda_{3}$ & $\Theta-1$ & $\Theta-1$ & $\Theta-1$ & $\Theta-1$ & $2 \Lambda$ & $2 \Lambda$ & $2 \Lambda$ & $2 \Lambda$ & $\mathcal{Z}$ \\
$-2 \Lambda_{3}$ & $-2 \Lambda_{3}$ & $\Theta+1$ & $\Theta+1$ & $\Theta+1$ & $\Theta+1$ & $2 \Lambda$ & $2 \Lambda$ & $2 \Lambda$ & $2 \Lambda$ & $\mathcal{Z}$ \\
\hline$\Theta$ & $\Theta$ & $\Theta$ & $\Theta$ & $\Theta$ & $\Theta$ & $\mathcal{Z}$ & $\mathcal{Z}$ & $\mathcal{Z}$ & $\mathcal{Z}$ & $\mathcal{Z}$ \\
$2 \Theta$ & $2 \Theta$ & $\mathcal{Z}$ & $\mathcal{Z}$ & $\mathcal{Z}$ & $\mathcal{Z}$ & $2 \Theta$ & $2 \Theta$ & $2 \Theta$ & $2 \Theta$ & $\mathcal{Z}$ \\
\hline$\Gamma$ & $\Gamma$ & $\Theta+1$ & $\Theta+1$ & $\Theta+1$ & $\Theta+1$ & $2 \Theta+2$ & $2 \Theta+2$ & $2 \Theta+2$ & $2 \Theta+2$ & $\mathcal{Z}$ \\
$-\Gamma$ & $-\Gamma$ & $\Theta-1$ & $\Theta-1$ & $\Theta-1$ & $\Theta-1$ & $2 \Theta-2$ & $2 \Theta-2$ & $2 \Theta-2$ & $2 \Theta-2$ & $\mathcal{Z}$ \\
$2 \Gamma$ & $2 \Gamma$ & $\Theta-1$ & $\Theta-1$ & $\Theta-1$ & $\Theta-1$ & $2 \Theta+2$ & $2 \Theta+2$ & $2 \Theta+2$ & $2 \Theta+2$ & $\mathcal{Z}$ \\
$-2 \Gamma$ & $-2 \Gamma$ & $\Theta+1$ & $\Theta+1$ & $\Theta+1$ & $\Theta+1$ & $2 \Theta-2$ & $2 \Theta-2$ & $2 \Theta-2$ & $2 \Theta-2$ & $\mathcal{Z}$ \\
\hline
\end{tabular}

Table 1: The Cayley table for the set $T_{17}$.

Since each element of $\lambda\left(C_{5}\right)$ can be uniquely written as the sum $\mathcal{L}+b$ for some $\mathcal{L} \in T_{17}$ and $b \in C_{5}$, the multiplication table for the semigroup $\lambda\left(C_{5}\right)$ can be recovered from the Cayley Table 1 for multiplication of the elements of the set $T_{17}$.

Now we are able to prove the main result of this subsection.

Theorem 2. $\operatorname{Aut}\left(\lambda\left(C_{5}\right)\right) \cong \operatorname{Aut}\left(C_{5}\right) \cong C_{4}$.

Proof. We identify the group Aut $\left(C_{5}\right)$ with the subgroup $\left\{\lambda \varphi: \varphi \in \operatorname{Aut}\left(C_{5}\right)\right\}$ of $\operatorname{Aut}\left(\lambda\left(C_{5}\right)\right)$. To see that $\operatorname{Aut}\left(\lambda\left(C_{5}\right)\right)=\operatorname{Aut}\left(C_{5}\right)$, it suffices to prove that an automorphism $\psi$ of $\lambda\left(C_{5}\right)$ is identity if its restriction $\psi \mid C_{5}$ is the identity automorphism of the group $C_{5}$. So, we assume that $\psi(x)=x$ for all $x \in C_{5}$. Since each element $\mathcal{A} \in \lambda\left(C_{5}\right)$ is of the form $\mathcal{A}=\mathcal{L}+x$ for some $\mathcal{L} \in T_{17}$ and $x \in C_{5}$, it suffices to prove that $\psi(\mathcal{L})=\mathcal{L}$ for every $\mathcal{L} \in T_{17}$.

Let us call a subset $A \subset \lambda\left(C_{5}\right) \psi$-invariant if $\psi(A)=A$. An element $a \in \lambda\left(C_{5}\right)$ is defined to be $\psi$-invariant if the singleton $\{a\}$ is $\psi$-invariant. Observe that for any $\psi$-invariant sets $A, B \subset \lambda\left(C_{5}\right)$ the set $A * B=\{a * b: a \in A, b \in B\}$ is $\psi$-invariant. In particular, the set $A+x$ is $\psi$-invariant for every $x \in C_{5}$.

Since $\psi$ is an automorphism of the semigroup $\lambda\left(C_{5}\right)$, the set $E\left(\lambda\left(C_{5}\right)\right)=\left\{\mathcal{U}, \mathcal{Z}, \Lambda_{4}, \Lambda, 2 \Lambda\right\}$ of idempotents of $\lambda\left(C_{5}\right)$ is $\psi$-invariant and $\psi \mid E\left(\lambda\left(C_{5}\right)\right)$ is an automorphism of the semilattice $E\left(\lambda\left(C_{5}\right)\right)$. Looking at the structure of the semilattice $E\left(\lambda\left(C_{5}\right)\right)$, we can see that its elements $\mathcal{Z}, \mathcal{U}$ and $\Lambda_{4}$ are $\psi$-invariant and hence $\psi(\Lambda) \in\{\Lambda, 2 \Lambda\}$.

Two cases are possible:

1) $\psi(\Lambda)=\Lambda$. In this case the following sets are $\psi$-invariant:

$\{\Lambda\}, \quad\{2 \Lambda\}, \quad \sqrt{\Lambda} \backslash\{\Lambda\}=\left\{\Delta, \Lambda_{3},-\Lambda_{3}\right\}, \sqrt{\{2 \Lambda\}} \backslash\{2 \Lambda\}=\left\{2 \Delta,-2 \Lambda_{2}, 2 \Lambda_{3}\right\}$, $\left\{2 \Delta,-2 \Lambda_{2}, 2 \Lambda_{3}\right\} *\{\Lambda\}=\{\Theta, \Theta-1, \Theta+1\}, \quad\left\{\Delta, \Lambda_{3},-\Lambda_{3}\right\} *\{2 \Lambda\}=\{2 \Theta, 2 \Theta+2,2 \Theta-2\}$. 
It follows that the set $\{\Theta, \Theta-1, \Theta+1\}+2=\{\Theta+2, \Theta+1, \Theta+3\}$ is $\psi$-invariant and so are the sets $\{\Theta+1\}=\{\Theta, \Theta-1, \Theta+1\} \cap\{\Theta+2, \Theta+1, \Theta+3\}$ and $\{\Theta\}$. By analogy we can prove that the element $2 \Theta$ is $\psi$-invariant.

Since the elements $\Lambda, 2 \Lambda, \Theta$ and $2 \Theta$ are $\psi$-invariant, the set

$$
\left\{\mathcal{L} \in \lambda\left(C_{5}\right): \mathcal{L} * \mathcal{L}=\Lambda, \mathcal{L} * \Lambda=\Lambda, \mathcal{L} * 2 \Lambda=2 \Theta\right\}=\{\Delta\}
$$

is $\psi$-invariant and hence the element $\Delta$ is $\psi$-invariant. By analogy we can prove that the element $2 \Delta, \Lambda_{3}, 2 \Lambda_{3},-\Lambda_{3},-2 \Lambda_{3}$ are $\psi$-invariant.

Since the elements $\mathcal{Z}, \Lambda, \Theta+1,2 \Theta+2$ are $\psi$-invariant, the set

$$
\left\{\mathcal{L} \in \lambda\left(C_{5}\right): \mathcal{L} * \mathcal{L}=\mathcal{Z}, \mathcal{L} * \Lambda=\Theta+1, \mathcal{L} * 2 \Lambda=2 \Theta+2\right\}=\{\Gamma\}
$$

is $\psi$-invariant. So, the element $\Gamma$ is $\psi$-invariant. By analogy we can prove that the elements $-\Gamma, 2 \Gamma,-2 \Gamma$ are $\psi$-invariant.

Therefore, $\psi(\mathcal{L})=\mathcal{L}$ for all $\mathcal{L} \in T_{17}$ and $\psi$ is the identity automorphism of $\lambda\left(C_{5}\right)$.

2) $\psi(\Lambda) \neq \Lambda$. Then $\psi(\Lambda)=2 \Lambda, \psi(2 \Lambda)=\Lambda$, and by the first case, $\psi \circ \psi$ is the identity automorphism of $\lambda\left(C_{5}\right)$. It follows that

$$
\begin{gathered}
\psi\left(\left\{\Delta, \Lambda_{3},-\Lambda_{3}\right\}\right)=\psi(\sqrt{\Lambda} \backslash\{\Lambda\})=\sqrt{2 \Lambda} \backslash\{2 \Lambda\}=\left\{2 \Delta, 2 \Lambda_{3},-2 \Lambda_{3}\right\}, \\
\psi(\{\Theta, \Theta-1, \Theta+1\})=\psi\left(\left\{2 \Delta, 2 \Lambda_{3},-2 \Lambda_{3}\right\} *\{\Lambda\}\right)=\left\{\Delta, \Lambda_{3},-\Lambda_{3}\right\} *\{2 \Lambda\}=\{2 \Theta, 2 \Theta+2,2 \Theta-2\} .
\end{gathered}
$$

Then $\psi(\{\Theta+1, \Theta, \Theta+2\})=\psi(\{\Theta, \Theta-1, \Theta+1\}+1)=\{2 \Theta, 2 \Theta+2,2 \Theta-2\}+1=$ $\{2 \Theta+1,2 \Theta-2,2 \Theta-1\}$ and hence $\psi(\{\Theta, \Theta+1\})=\psi(\{\Theta, \Theta-1, \Theta+1\} \cap\{\Theta+1, \Theta, \Theta+2\})=$ $\{2 \Theta, 2 \Theta+2,2 \Theta-2\} \cap\{2 \Theta+1,2 \Theta-2,2 \Theta-1\}=\{2 \Theta-2\}$, which contradicts the bijectivity of $\psi$. So, the case $\psi(\Lambda) \neq \Lambda$ is impossible and by the first case, $\psi$ is the identity automorphism of $\lambda\left(C_{5}\right)$. Therefore, $\operatorname{Aut}\left(\lambda\left(C_{5}\right)\right)=\operatorname{Aut}\left(C_{5}\right) \cong C_{4}$.

2.6. The final table. We summarize the obtained results on the automorphism groups Aut $(\lambda(G))$ of superextensions of groups $G$ of cardinality $|G| \leq 5$ in the following table:

\begin{tabular}{|c|cccccc|}
\hline$G$ & $C_{1}$ & $C_{2}$ & $C_{3}$ & $C_{4}$ & $C_{2} \times C_{2}$ & $C_{5}$ \\
\hline $\operatorname{Aut}(G)$ & $C_{1}$ & $C_{1}$ & $C_{2}$ & $C_{2}$ & $S_{3}$ & $C_{4}$ \\
\hline $\operatorname{Aut}(\lambda(G))$ & $C_{1}$ & $C_{1}$ & $C_{2}$ & $C_{2} \times C_{2}$ & $S_{4}$ & $C_{4}$ \\
\hline
\end{tabular}

Analyzing the entries of this table, we can ask the following question.

Problem 1. Is Aut $(\lambda(G))$ isomorphic to $\operatorname{Aut}(G)$ for a finite (cyclic) group $G$ of odd order?

\section{REFERENCES}

1. T. Banakh, V. Gavrylkiv, Algebra in superextension of groups, II: cancelativity and centers, Algebra Discrete Math., 4 (2008), 1-14.

2. T. Banakh, V. Gavrylkiv, Algebra in superextension of groups: minimal left ideals, Mat. Stud., 31 (2009), №2, 142-148.

3. T. Banakh, V. Gavrylkiv, Extending binary operations to functor-spaces, Carpathian Math. Publ., 1 (2009), №2, 113-126. 
4. T. Banakh, V. Gavrylkiv, Algebra in the superextensions of twinic groups, Dissertationes Math., 473 (2010), 3-74.

5. T. Banakh, V. Gavrylkiv, Algebra in superextensions of semilattices, Algebra Discrete Math., 13 (2012), №1, 26-42.

6. T. Banakh, V. Gavrylkiv, Algebra in superextensions of inverse semigroups, Algebra Discrete Math., 13 (2012), №2, 147-168.

7. T. Banakh, V. Gavrylkiv, Characterizing semigroups with commutative superextensions, Algebra Discrete Math., 17 (2014), №2, 161-192.

8. T. Banakh, V. Gavrylkiv, On structure of the semigroups of k-linked upfamilies on groups, AsianEuropean J. Math., 10 (2017), №4, 15 p.

9. T. Banakh, V. Gavrylkiv, O. Nykyforchyn, Algebra in superextensions of groups, I: zeros and commutativity, Algebra Discrete Math., 3 (2008), 1-29.

10. A.E. Brouwer, C.F. Mills, W.H. Mills, A. Verbeek, Counting families of mutually intesecting sets, Electron. J. Combin., 20 (2013), №2, 8 p.

11. V. Gavrylkiv, The spaces of inclusion hyperspaces over noncompact spaces, Mat. Stud., 28 (2007), №1, 92-110.

12. V. Gavrylkiv, Right-topological semigroup operations on inclusion hyperspaces, Mat. Stud., 29 (2008), №1, 18-34.

13. V. Gavrylkiv, On representation of semigroups of inclusion hyperspaces, Carpathian Math. Publ., 2 (2010), №1, 24-34.

14. V. Gavrylkiv, Superextensions of cyclic semigroups, Carpathian Math. Publ., 5 (2013), №1, 36-43.

15. V. Gavrylkiv, Semigroups of centered upfamilies on finite monogenic semigroups, J. Algebra, Number Theory: Adv. App., 16 (2016), №2, 71-84.

16. V. Gavrylkiv, Semigroups of centered upfamilies on groups, Lobachevskii J. Math., 38 (2017), №3, 420428.

17. V. Gavrylkiv, Superextensions of three-element semigroups, Carpathian Math. Publ., 9 (2017), №1, 2836.

18. V. Gavrylkiv, On the automorphism group of the superextension of a semigroup, Mat. Stud., 48 (2017), №1, 3-13.

19. N. Hindman, D. Strauss, Algebra in the Stone-Čech compactification, de Gruyter, Berlin, New York, 1998.

20. J.M. Howie, Fundamentals of semigroup theory, The Clarendon Press Oxford University Press, New York, 1995.

21. J. van Mill, Supercompactness and Wallman spaces, Mathematical Centre Tracts, V.85 Amsterdam, 1977.

22. D. Robinson, A course in the theory of groups, Graduate Texts in Mathematics, V.80 (Springer-Verlag, New York, 1996).

23. A. Teleiko, M. Zarichnyi, Categorical Topology of Compact Hausdofff Spaces, V.5, VNTL, Lviv, 1999.

24. A. Verbeek, Superextensions of topological spaces, Mathematical Centre Tracts, V.41, Amsterdam, 1972.

Ivan Franko National University of Lviv, Ukraine, and Institute of Mathematics, Jan Kochanowski University in Kielce, Poland t.o.banakh@gmail.com

Faculty of Mathematics and Computer Science Vasyl Stefanyk Precarpathian National University vgavrylkiv@gmail.com 